\title{
Sine-Gordon kink-antikink generation on spatially periodic potentials
}

\author{
Rainer Scharf, Yuri S. Kivshar, ${ }^{*}$ Angel Sánchez, ${ }^{\dagger}$ and Alan R. Bishop \\ Theoretical Division and Center for Nonlinear Studies, Los Alamos National Laboratory, Los Alamos, New Mexico 87545
}

(Received 4 November 1991)

\begin{abstract}
A spatially periodic perturbation can lead to a breakup of large-amplitude sine-Gordon breathers into kink and antikink solutions, each oscillating around a minimum of the perturbing potential. This behavior can be understood by studying the effective potential experienced by the breather (bound kink-antikink) or the (free) kink-antikink solution as long as kink and antikink are sufficiently far apart. The resulting kinks and antikinks move independently and nearly radiationlessly in the presence of the perturbation and can travel arbitrarily far for sufficiently large initial kinetic energy. Upon interacting with each other they are strongly affected by the perturbation, lose energy by radiating, and can end in a bound state having the character of a distorted breather.
\end{abstract}

PACS numbers: $03.40 . \mathrm{Kf}, 42.20 .-y, 61.70 . \mathrm{Ga}$

Adding spatial disorder to completely integrable nonlinear dynamics like the one governed by the nonlinear Schrödinger (NLS) equation or the sine-Gordon (SG) equation leads to a variety of novel effects having practical relevance [1]. Competition of the length scales introduced by the perturbation and by the nonlinearity is one example we have studied recently [2]. If these length scales are very different from each other the perturbed dynamics can support solitonlike or breatherlike excitations. The motion of these excitations can be described by a collective variable approach $[3,4]$. On the other hand, if the length scales are comparable localized excitations break up or dissipate into radiation even for relatively small strength of the perturbation. This behavior has been observed in the case of the SG equation [2] as well as for the NLS equation [5].

Another class of effects induced by spatially periodic perturbations can be observed in dynamics which allow for topological solitons like the SG equation. Although very many perturbed $S G$ problems have been considered in the literature (see, e.g., $[6,7]$ for reviews), to our best knowledge, only Mkrtchyan and Shmidt [8] and Malomed and Tribelsky [9] have studied the motion of SG kinks under the influence of a cosine potential. In this case kinks (and antikinks) seem to move radiationlessly below a certain velocity threshold. The question arises whether kinks and antikinks also interact radiationlessly under these circumstances. Finally it is interesting to see under which conditions a breather can break up into a kinkantikink $(K-\bar{K})$ pair under the perturbation [2].

In this Rapid Communication we address the question of how large-amplitude SG breathers behave under the influence of spatially periodic potentials. These potentials include the effects of discreteness as a particular case; they also can be relevant to understand the interaction of domain wall excitations in discrete solid-state and materials science problems governed by SG-like equations of motion, for example, dislocations in a crystal, walls in ferroelectrics or ferromagnets, or discommensurations in superlattices. A breather can be interpreted as a bound $K-\bar{K}$ state (see a detailed account of the physics of breathers and kinks in [10], for instance). Under a sufficiently strong potential these virtual kinks and antikinks can become independent of each other. The opposite effect of binding previously free kinks and antikinks in a breatherlike excitation will also be addressed.

The perturbed SG equation we investigate is

$$
u_{t t}-u_{x x}+[1+\epsilon \cos (k x)] \sin u=0 .
$$

This equation of motion is generated by the Hamiltonian (see [6])

$$
H=\int_{-\infty}^{+\infty} d x\left\{\frac{1}{2} u_{t}^{2}+\frac{1}{2} u_{x}^{2}+[1+\epsilon \cos (k x)](1-\cos u)\right\}
$$

We will use two different kinds of exact solutions of the unperturbed $S G$ equation $(\epsilon=0)$ as initial conditions: breathers and $K-\bar{K}$ solutions. The solution for a breather at rest has the form

$$
u^{\mathrm{br}}(x, t)=4 \tan ^{-1}\left(\tan \mu \frac{\sin \left[\left(t-t_{0}\right) \cos \mu\right]}{\cosh \left[\left(x-x_{0}\right) \sin \mu\right]}\right) .
$$

It is most important to realize that $\mu$ is the parameter governing the breather shape and size. As $\mu \rightarrow 0$, the breather becomes shallower, its frequency, given by $\omega_{\mathrm{br}}=$ $\cos \mu$, grows, and it can be effectively described by a NLS equation [11]. On the other hand, when $\mu \rightarrow \pi$, the breather frequency goes to zero and it is actually very close to a $K-\bar{K}$ pair.

As for the $K-\bar{K}$ solution, its expression at rest is given by

$$
u^{K \bar{K}}(x, t)=4 \tan ^{-1}\left(v^{-1} \frac{\sinh \left[\gamma v\left(t-t_{0}\right)\right]}{\cosh \left[\gamma\left(x-x_{0}\right)\right]}\right),
$$

with $v^{2}<1, \gamma \equiv\left(1-v^{2}\right)^{-1 / 2}$. Equation (4) can be obtained as well by analytic continuation (see, e.g., [12]) of Eq. (3) for the breather, letting $\mu=\pi / 2+i W$, with 
$\exp W=\gamma(1+v)$. Equations (3) and (4) can be derived following standard methods $[10,11]$.

The energy of an unperturbed breather at rest, as obtained from Eq. (2) evaluated for Eq. (3), is $E_{0}^{\mathrm{br}}=$ $16 \sin \mu$, whereas the same computation for an unperturbed $K-\bar{K}$ solution with its center of mass at rest is $E_{0}^{K \widetilde{K}}=16 \gamma$. In the absence of any perturbation the energies for breathers and $K-\bar{K}$-solutions at rest obviously fulfill $E_{0}^{\text {br }}<16<E_{0}^{K \bar{K}}$. The perturbation may shift this borderline between breathers and $K-\bar{K}$ solutions. Of relevance for our analysis will be the potential energy of an excitation in the absence of any perturbation:

$$
E_{\mathrm{pot}}^{0}=\int_{-\infty}^{+\infty} d x\left(\frac{1}{2} u_{x}^{2}+1-\cos u\right) .
$$

The amount by which the total energy is changed in the presence of the perturbation is given by

$$
V_{\mathrm{eff}}=\int_{-\infty}^{+\infty} d x \epsilon \cos (k x)(1-\cos u)
$$

For a breather at rest with $t_{0}=0$ one calculates

$$
\begin{aligned}
E_{\mathrm{pot}}^{0}= & 8 \sin \mu+\frac{8 \tanh ^{2} z}{\sin \mu} \\
-\frac{8 z \sin \mu\left(1-\cot ^{2} \mu \sinh ^{2} z\right)}{\sinh z \cosh ^{3} z} & \\
V_{\mathrm{eff}}^{\mathrm{br}}\left(x_{0}, z\right)= & \frac{4 \pi \epsilon \sinh z \cos \left(k x_{0}\right)}{\sin \mu \cosh z \sinh (K \pi / 2)} \\
& \times\left(\frac{\sin (K z)}{\cosh z}+K \cos (K z) \sinh z\right)
\end{aligned}
$$

where $K \equiv k / \sin \mu$ and $z$, defined by $\sinh z \equiv$ $\tan \mu \sin (t \cos \mu)$, is a measure of the distance between the kink and the antikink bound in the breather.

For a $K-\bar{K}$ solution at rest with $t_{0}=0$ one finds correspondingly

$$
\begin{aligned}
E_{\mathrm{pot}}^{0}=8 \gamma+ & \frac{8 \tanh ^{2} z}{\gamma}-\frac{8 \gamma z\left(1+v^{2} \sinh ^{2} z\right)}{\sinh z \cosh ^{3} z}, \\
V_{\mathrm{eff}}^{K \bar{K}}\left(x_{0}, z\right)= & \frac{4 \pi \epsilon \sinh z \cos \left(k x_{0}\right)}{\gamma \cosh ^{2} z \sinh (K \pi / 2)} \\
& \times\left(\frac{\sin (K z)}{\cosh z}+K \cos (K z) \sinh z\right),
\end{aligned}
$$

where $K \equiv k / \gamma$ and $z$, defined by $\sinh z \equiv v^{-1} \sinh (\gamma v t)$, is a measure of the distance between the kink and the antikink.

The effective potential $V_{\text {eff }}$ depends on the distance between the (virtual) kink and antikink $(z)$ and on the center of mass $x_{0}$ of the $K-\bar{K}$ solution or the breather. For nonrelativistic velocities $(\gamma \approx 1) V_{\text {eff }}\left(x_{0}, z\right)$ can be used to calculate the influence of the potential on the motion of the center of the excitation as well as on the relative distance between kink and antikink in an adiabatic approximation, assuming that the parameter $z$ can be considered as a second collective variable. This and the effect of the coupling between the translational and the internal degrees of freedom of the breather or the $K$ $\bar{K}$ solution will be addressed in [13]. Here we restrict the analysis to breathers and $K-\bar{K}$ solutions at rest.

A breather is a bound state solution of the $\mathrm{SG}$ equation which oscillates around $u=0$ with a period $T=$ $2 \pi / \cos \mu$. For sufficiently strong perturbations the total potential energy $E_{\text {pot }}=E_{\text {pot }}^{\overline{0}}+V_{\text {eff }}$ can have other minima besides $u \equiv 0$ around which the solution can oscillate.

In Fig. 1 we show the potential energy $E_{\text {pot }}$ for breathers (a) and $K-\bar{K}$ solutions (b). We set $x_{0}=0$, $\epsilon=0.1$, and $k=\pi / 2$. Figure 1 (a) refers to a range of breathers each having reached their maximum amplitude and spatial extension and therefore their kinetic energy vanishes $(t=\pi / 2 \cos \mu)$. For $\mu$ approaching $\pi / 2$ the breathers become increasingly extended and $V_{\text {eff }}$ oscillates increasingly fast, whereas for $\mu \rightarrow 0$ the potential energy goes to 0 rapidly. For $\epsilon=0 E_{\text {pot }}$ would be a monotonous function with only one minimum at $\mu=0$. As can be seen from Fig. 1(a), when $\epsilon \neq 0$ new minima and maxima appear.
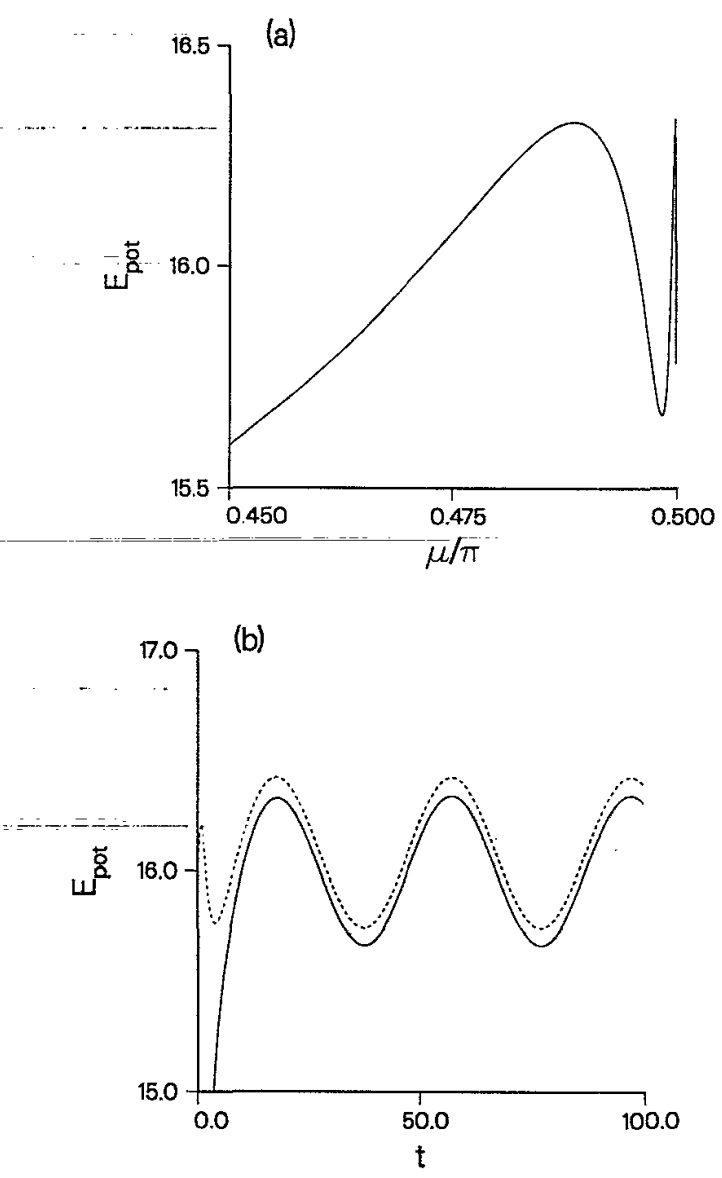

FIG. 1. The potential energy for $\epsilon=0.1, k=\pi / 2, x_{0}=0$. (a) Different breathers with $t=\pi / 2 \cos \mu$. The parameters $t$ and $z$ are related by $\sin (t \cos \mu)=\cot \mu \sinh z$. (b) $K-\bar{K}$ solutions with $v=0.1$ for $0<t<100$. The parameters $t$ and $z$ are related by $\sinh (\gamma v t)=v \sinh z$. The dashed curve shows the total energy $E=E_{\text {kin }}+E_{\text {pot }}$. Both curves are symmetric around $t=0$. A full explanation is given in the text. 
Starting with a breather of vanishing kinetic energy at a maximum of $E_{\text {pot }}(\mu)$ and allowing for an infinitesimal change in $\mu$ amounts to testing the stability of the breathers against slight changes of its size. Therefore we expect that for $\mu<\mu_{1} \approx 1.5355$ [left side of the first maximum in Fig. 1(a)] the breather still behaves breatherlike, oscillating around $u \equiv 0$, although with a different period $T$. For $\mu>\mu_{1}$ the breather will split into kink and antikink. As the second maximum at $\mu_{2} \approx 1.57$ is slightly higher than the first, kink and antikink will oscillate independently around the minimum of $E_{\text {pot }}(\mu)$ between $\mu_{1}$ and $\mu_{2}$. Figure 2 shows the results of numerical simulations of Eq. (1) which confirm the predictions in detail. It is interesting to note that there is no radiation visible as long as kink and antikink are sufficiently far apart. In striking contrast to this the amount of radiation increases drastically as kink and antikink approach each other and the solution evolves through $u \equiv 0$.

Figure 1(b) shows the potential energy $E_{\text {pot }}$ for a $K-\bar{K}$ solution with small relative velocity $(v=0.1)$ for a time interval. In addition, the dashed curve indicates the total energy for each initial condition. We can see that the minima of $E_{\text {pot }}$ are sufficiently deep to allow for bound states. Starting on one of the maxima with negative ve-
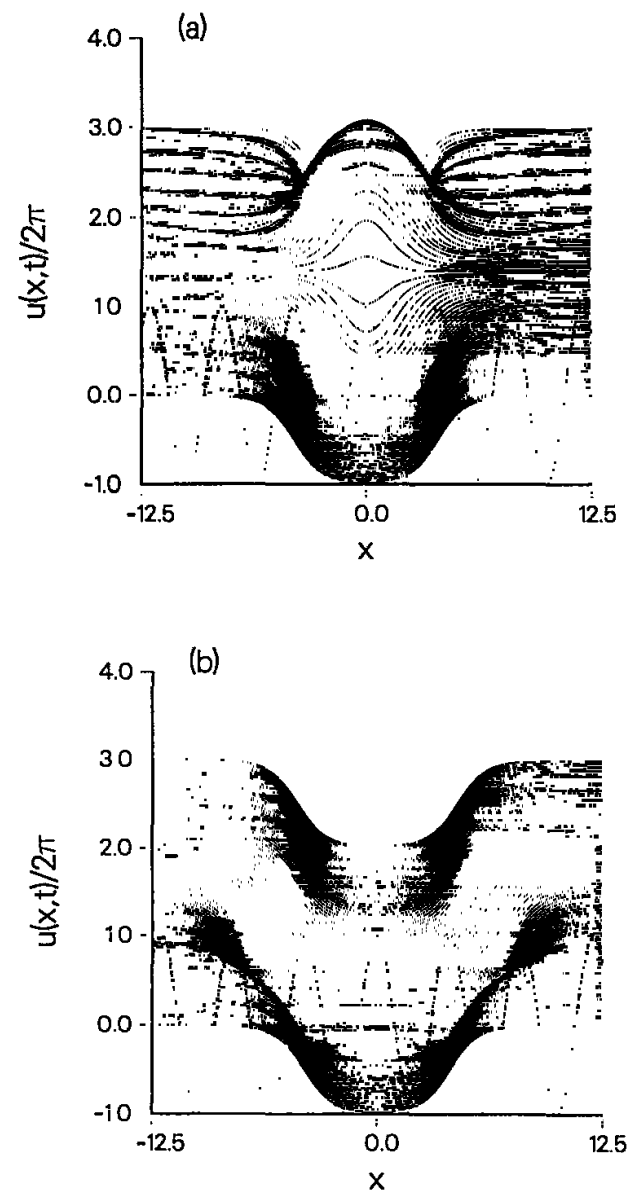

FIG. 2. Numerical simulation of Eq. (1) with the parameters $\epsilon=0.1$ and $k=\pi / 2$ for a breather initial condition [Eq. (3)] with $x_{0}=t_{0}=0, t=\pi / 2 \cos \mu$. (a) $\mu=1.535$; (b) $\mu=1.536$. locity $v$ separates the kink and the antikink. Starting with positive $v$ leads to a collision and $E_{\text {pot }}$ drops rapidly to 0 as the relative distance vanishes.

For example, inspecting Fig. 1(b) for initial conditions with $|t|>22$ we predict oscillatory behavior of kink and antikink independently around the second minimum in Fig. 1(b) at $|t| \approx 37$. Starting the $K-\bar{K}$ solution on the second maximum at $t=-60 \mathrm{kink}$ and antikink have sufficiently large kinetic energy to collide. In Fig. 3 we show the corresponding numerical simulations of Eq. (1). Again these results confirm our predictions. Concerning radiative effects we again find that as long as kink and antikink are sufficiently far apart radiative losses are negligible. But as kink and antikink collide [Fig. 3(b)] these losses become so important that they lead to a trapping of the kink and antikink around the minimum at $u \equiv 0$. This breatherlike trapped state continues to radiate. Starting with a larger relative velocity (for example, $v=0.2$ for $\epsilon=0.1$ and $k=\pi / 2$ ) kink and antikink will separate again after the collision.

In conclusion we found that simple energy considerations leading to Eqs. (7) and (8) allow for a prediction of the behavior of breathers or $K-\bar{K}$ solutions at rest under the influence of a spatially periodic pertur-
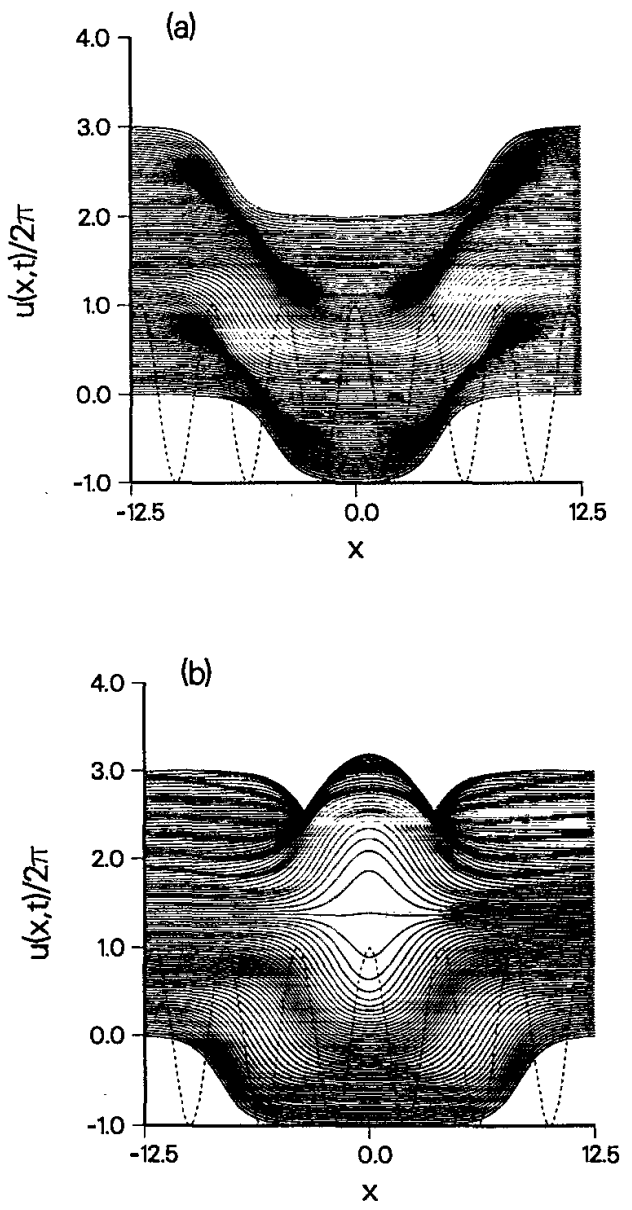

FIG. 3. Numerical simulation of Eq. (1) with the parameters $\epsilon=0.1$ and $k=\pi / 2$ for a breather initial condition [Eq. (4)] with $x_{0}=t_{0}=0, v=0.1$. (a) $t=-23$; (b) $t=-60$. 
bation. Our results also explain the numerical findings concerning breather breakup in [2]. In addition, we found that radiative effects, which play an important role for strongly interacting kinks and antikinks, are negligible for isolated kinks with small velocities. These radiative aspects will be addressed in a future publication.

\section{ACKNOWLEDGMENTS}

We acknowledge support by the U.S. Department of Energy. A. S. is partially supported by the Comision Interministerial de Ciencia y Tecnologia (CICyT) of Spain under Grant No. MAT90-0544.
* Present address: Institut für Theoretische Physik I, Universität Düsseldorf, D-4000 Düsseldorf 1, Germany. Permanent address: Institute for Low Temperature Physics and Engineering, Academy of Sciences of Ukraine, Kharkov 310164, Ukraine.

$\dagger$ Permanent address: -Departamento de Física Teórica I, Facultad de Ciencias Físicas, Universidad Complutense, E-28040 Madrid, Spain.

[1] Disorder and Nonlinearity, edited by A. R. Bishop, D. K. Campbell, and St. Pnevmatikos, Springer Proceedings in Physics Vol. 39 (Springer-Verlag, Berlin, 1989); Nonlinearity with Disorder, edited by F. Kh. Abdullaev, A. R. Bishop, and St. Pnevmatikos, Springer Proceedings in Physics (Springer-Verlag, Berlin, in press).

[2] A. Sánchez, R. Scharf, L. Vázquez, and A. R. Bishop, Phys. Rev. A 45, 6031 (1992).

[3] R. Boesch and C. Willis, Phys. Rep. (to be published).

[4] R. Boesch and M. Peyrard, Phys. Rev. B 43, 8491 (1991).

[5] R. Scharf and A. R. Bishop (unpublished).
[6] Yu. S. Kivshar and B. A. Malomed, Rev. Mod. Phys. 61, 763 (1989).

[7] A. Sánchez and L. Vázquez, Int. J. Mod. Phys. B 5, 2825 (1991)

[8] G. S. Mkrtchyan and V. V. Shmidt, Solid State Commun. 30, 791 (1979).

[9] B. A. Malomed, in Solitons and Applications, edited by V. G. Makhankov, V. K. Fedyanin, and O. K. Pashaev (World Scientific, Singapore, 1990); B. A. Malomed and M. I. Tribelsky, Phys. Rev. B 41, 11271 (1990).

[10] G. L. Lamb, Elements of Soliton Theory (Wiley, New York, 1980); R. K. Dodd, J. C. Eilbeck, J. D. Gibbon, and H. C. Morris, Solitons and Nonlinear Wave Equations (Academic, London, 1982).

[11] A. C. Newell, Solitons in Mathematics and Physics (SIAM, Philadelphia, 1985).

[12] R. V. Churchill, Complex Variables and Applications (McGraw-Hill, New York, 1960), pp. 259-268.

[13] Yu. S. Kivshar and R. Scharf (unpublished). 